\title{
Rule, not exclusion: formation of dichlorine-containing supramolecular complexes with chlorometalates (IV)
}

Andrey N. Usoltsev, Nikita A. Korobeynikov, Boris A. Kolesov, Alexander S. Novikov, Denis G. Samsonenko, Vladimir P. Fedin, Maxim N. Sokolov and Sergey A. Adonin*

Powder X-ray diffractometry. XRD analysis of polycrystals was performed on Shimadzu XRD7000 diffractometer (CuK-alpha radiation, $\mathrm{Ni}$ - filter, linear One Sight detector, $5-50^{\circ} 2 \theta$ range, $0.0143^{\circ} 2 \theta$ step, 2 s per step). A polycrystalline sample was slightly ground with hexane in an agate mortar, and the resulting suspension was deposited on the polished side of a standard quartz sample holder, and a smooth thin layer being formed after drying. Plotting of PXRD patterns and data treatment was performed using X'Pert Plus software.

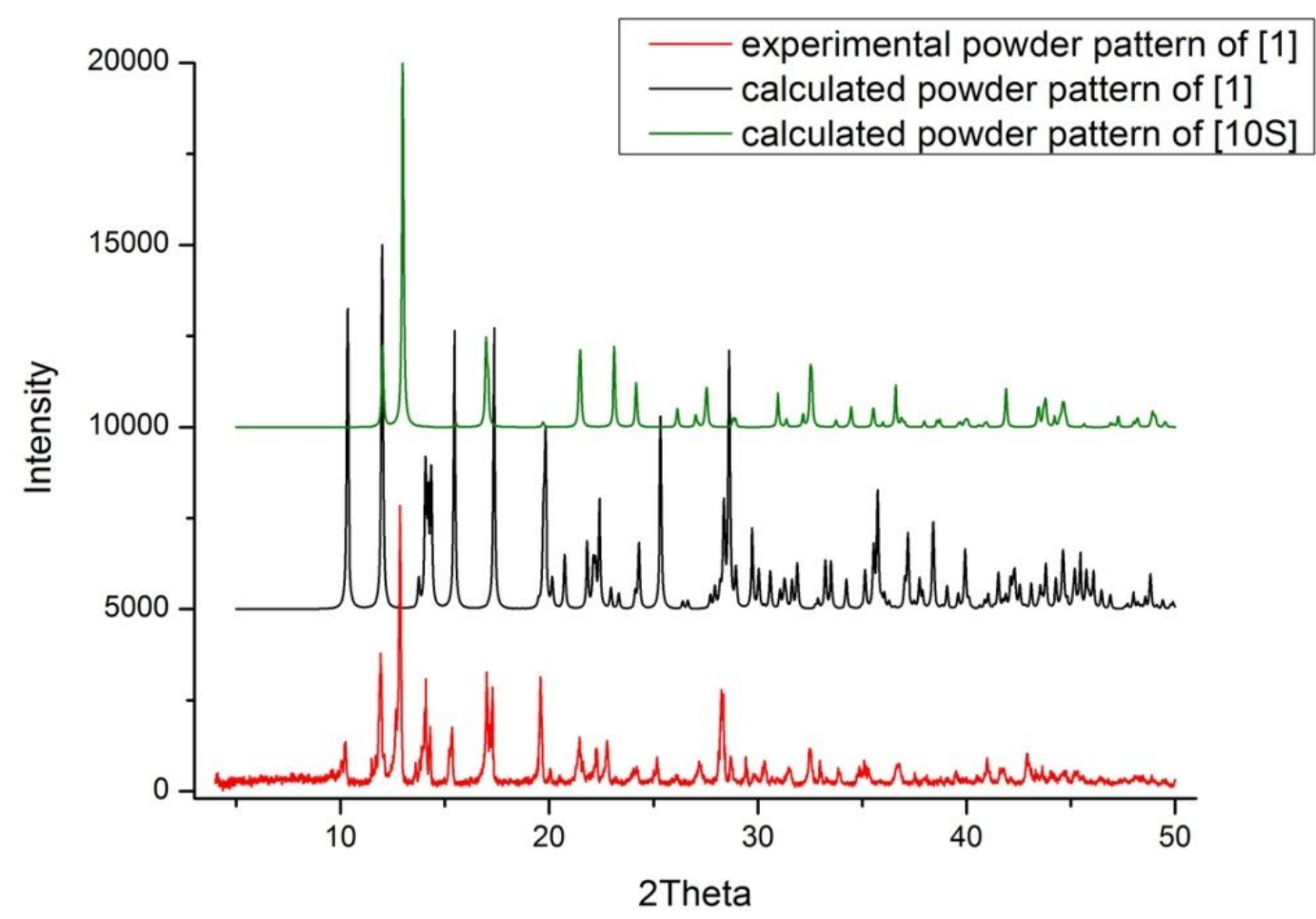

Figure S1. Comparison of experimental and calculated PXRD patterns (1 and 10S) 


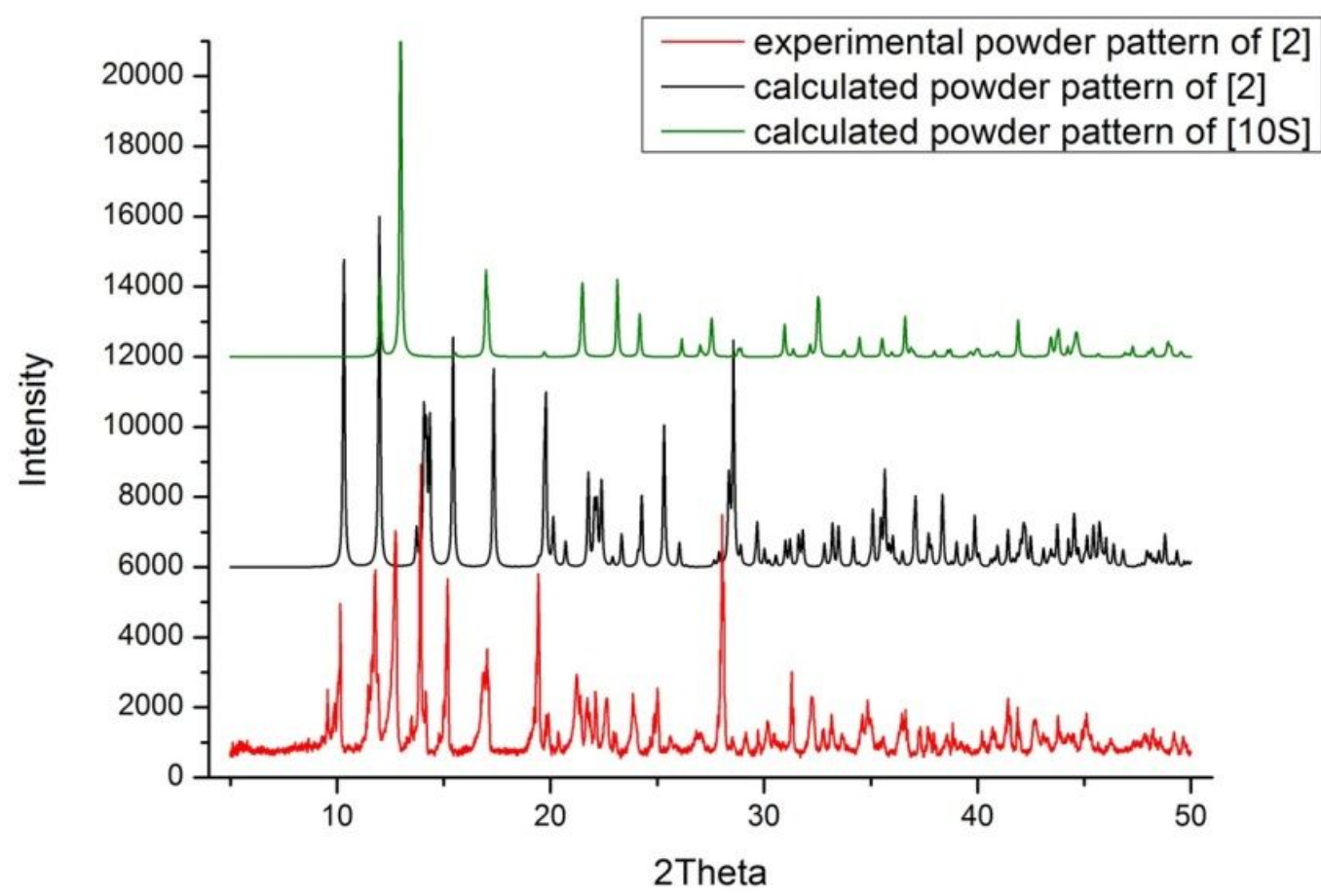

Figure S2. Comparison of experimental and calculated PXRD patterns ( 2 and 10 S) 


\section{S-3}

Table S1. Crystal data and structure refinement for 1S-10S.

\begin{tabular}{|c|c|c|c|c|c|c|c|c|c|c|}
\hline $\begin{array}{l}\text { Identification } \\
\text { code }\end{array}$ & $1 S$ & $2 S$ & 35 & $4 S$ & $5 S$ & $6 S$ & $7 S$ & $8 S$ & $9 \mathrm{~S}$ & $10 S$ \\
\hline $\begin{array}{l}\text { Empirical } \\
\text { formula }\end{array}$ & $\mathrm{C}_{18} \mathrm{H}_{28} \mathrm{Cl}_{6} \mathrm{~N}_{2} \mathrm{~Pb}$ & $\mathrm{C}_{10} \mathrm{H}_{12} \mathrm{Cl}_{6} \mathrm{~N}_{2} \mathrm{~Pb}$ & $\mathrm{C}_{12} \mathrm{H}_{16} \mathrm{Cl}_{6} \mathrm{~N}_{2} \mathrm{~Pb}$ & $\mathrm{C}_{12} \mathrm{H}_{16} \mathrm{Cl}_{6} \mathrm{~N}_{2} \mathrm{~Pb}$ & $\mathrm{C}_{12} \mathrm{H}_{16} \mathrm{Cl}_{6} \mathrm{~N}_{2} \mathrm{~Pb}$ & $\mathrm{C}_{10} \mathrm{H}_{10} \mathrm{Cl}_{8} \mathrm{~N}_{2} \mathrm{~Pb}$ & $\mathrm{C}_{10} \mathrm{H}_{12} \mathrm{Cl}_{8} \mathrm{~N}_{2} \mathrm{OPb}$ & $\mathrm{C}_{10} \mathrm{H}_{10} \mathrm{Br}_{2} \mathrm{Cl}_{6} \mathrm{~N}_{2} \mathrm{~Pb}$ & $\mathrm{C}_{10} \mathrm{H}_{12} \mathrm{Cl}_{10} \mathrm{~N}_{2} \mathrm{O}_{2} \mathrm{~Pb}$ & $\mathrm{C}_{12} \mathrm{H}_{16} \mathrm{Cl}_{6} \mathrm{~N}_{2} \mathrm{Sn}$ \\
\hline$M, \mathrm{~g} / \mathrm{mol}$ & 692.31 & 580.11 & 608.16 & 608.16 & 608.16 & 648.99 & 667.01 & 737.91 & 753.91 & 519.66 \\
\hline$T, \mathrm{~K}$ & $140(2)$ & $140(2)$ & $150(2)$ & $150(2)$ & $140(2)$ & $150(2)$ & $150(2)$ & $140(2)$ & $150(2)$ & $150(2)$ \\
\hline Crystal system & Monoclinic & Triclinic & Triclinic & Monoclinic & Monoclinic & Monoclinic & Triclinic & Monoclinic & Triclinic & Orthorhombic \\
\hline Space group & $P 2_{1} / c$ & $P-1$ & $P-1$ & $12 / a$ & $P 2_{1} / c$ & $P 2_{1} / n$ & $P-1$ & $P 2_{1} / n$ & $P-1$ & Pnnm \\
\hline$a, \AA$ & $8.5720(5)$ & $7.2209(4)$ & $7.0810(3)$ & $13.4427(6)$ & $8.6955(6)$ & $8.9212(7)$ & $8.2426(4)$ & $9.0049(7)$ & $7.2575(5)$ & $9.0128(5)$ \\
\hline$b, \AA$ & $10.3168(5)$ & 7.9913(4) & $8.1989(3)$ & $9.4265(4)$ & $16.7408(9)$ & $10.6563(8)$ & $11.3614(5)$ & $10.7231(8)$ & $8.2643(4)$ & $10.3802(5)$ \\
\hline$c, \AA$ & $14.0148(8)$ & $8.0415(4)$ & $8.8054(4)$ & $15.2827(6)$ & $7.1532(5)$ & $10.5893(8)$ & $11.5098(5)$ & $10.5890(8)$ & $9.9957(5)$ & $10.4374(8$ \\
\hline$\alpha$, deg. & 90 & $82.736(4)$ & $75.091(4)$ & 90 & 90 & 90 & $79.227(4)$ & 90 & $89.127(4)$ & 90 \\
\hline$\beta$, deg. & $104.683(6)$ & $65.278(5)$ & $70.924(4)$ & $97.674(4)$ & $113.551(9)$ & 109.945(8) & $75.814(4)$ & 110.099(9) & $73.150(5)$ & 90 \\
\hline$\gamma$, deg. & 90 & $84.059(5)$ & $83.509(4)$ & 90 & 90 & 90 & $89.805(4)$ & 90 & $75.613(5)$ & 90 \\
\hline$V, \AA^{3}$ & $1198.93(12)$ & $417.46(4)$ & $466.64(4)$ & 1919.24(14) & $954.55(12)$ & $946.31(13)$ & $1025.56(8)$ & $960.21(14)$ & $554.75(6)$ & $976.47(10)$ \\
\hline$Z$ & 2 & 1 & 1 & 4 & 2 & 2 & 2 & 2 & 1 & 2 \\
\hline$D$ (calc.), $\mathrm{g} / \mathrm{cm}^{3}$ & 1.918 & 2.308 & 2.164 & 2.105 & 2.116 & 2.278 & 2.160 & 2.552 & 2.257 & 1.767 \\
\hline
\end{tabular}




\section{S-4}

\begin{tabular}{|c|c|c|c|c|c|c|c|c|c|c|}
\hline$\mu, \mathrm{mm}^{-1}$ & 7.712 & 11.050 & 9.891 & 9.619 & 9.671 & 10.036 & 9.267 & 13.774 & 8.816 & 2.123 \\
\hline$F(000)$ & 668 & 270 & 286 & 1144 & 572 & 604 & 624 & 676 & 354 & 508 \\
\hline Crystal size, mm & $\begin{array}{l}0.23 \times 0.17 \times \\
0.09\end{array}$ & $\begin{array}{l}0.23 \times 0.19 \times \\
0.07\end{array}$ & $\begin{array}{l}0.40 \times 0.25 \times \\
0.14\end{array}$ & $\begin{array}{l}0.40 \times 0.31 \times \\
0.25\end{array}$ & $\begin{array}{l}0.32 \times 0.22 \times \\
0.09\end{array}$ & $\begin{array}{l}0.26 \times 0.24 \times \\
0.12\end{array}$ & $\begin{array}{l}0.36 \times 0.17 \times \\
0.09\end{array}$ & $\begin{array}{l}0.34 \times 0.22 \times \\
0.12\end{array}$ & $\begin{array}{l}0.24 \times 0.18 \times \\
0.12\end{array}$ & $\begin{array}{l}0.26 \times 0.10 \times \\
0.09\end{array}$ \\
\hline $\begin{array}{l}\theta \text { range for data } \\
\text { collection, deg. }\end{array}$ & $2.46-28.88$ & $2.57-28.85$ & $2.52-28.91$ & $2.54-28.97$ & $2.83-28.94$ & $2.59-29.00$ & $2.34-25.68$ & $2.57-28.87$ & $2.13-28.96$ & $2.77-25.68$ \\
\hline Index ranges & $\begin{array}{l}-10 \leq h \leq 11 \\
-13 \leq k \leq 13 \\
-18 \leq l \leq 18\end{array}$ & $\begin{array}{l}-9 \leq h \leq 8 \\
-10 \leq k \leq 6 \\
-10 \leq l \leq 9\end{array}$ & $\begin{array}{l}-8 \leq h \leq 9 \\
-9 \leq k \leq 11 \\
-7 \leq l \leq 10\end{array}$ & $\begin{array}{l}-12 \leq h \leq 17 \\
-12 \leq k \leq 11 \\
-20 \leq l \leq 20\end{array}$ & $\begin{array}{l}-11 \leq h \leq 11 \\
-22 \leq k \leq 22 \\
-9 \leq l \leq 9\end{array}$ & $\begin{array}{l}-7 \leq h \leq 11 \\
-11 \leq k \leq 13 \\
-13 \leq l \leq 10\end{array}$ & $\begin{array}{l}-10 \leq h \leq 10 \\
-8 \leq k \leq 13 \\
-14 \leq l \leq 13\end{array}$ & $\begin{array}{l}-11 \leq h \leq 9 \\
-13 \leq k \leq 14 \\
-13 \leq l \leq 13\end{array}$ & $\begin{array}{l}-9 \leq h \leq 9 \\
-11 \leq k \leq 9 \\
-12 \leq l \leq 13\end{array}$ & $\begin{array}{l}-10 \leq h \leq 10 \\
-12 \leq k \leq 10 \\
-8 \leq l \leq 12\end{array}$ \\
\hline $\begin{array}{l}\text { Reflections } \\
\text { collected / } \\
\text { independent }\end{array}$ & $5923 / 2649$ & 3166 / 1831 & $3626 / 2026$ & 4792 / 2149 & 5067 / 2134 & 4458 / 2090 & 7339 / 3864 & 4577 / 2117 & 4292 / 2436 & 2557 / 975 \\
\hline$R_{\text {int }}$ & 0.0281 & 0.0384 & 0.0390 & 0.0238 & 0.0194 & 0.0283 & 0.0443 & 0.0256 & 0.0321 & 0.0332 \\
\hline $\begin{array}{l}\text { Reflections with } \\
I>2 \sigma(I)\end{array}$ & 2132 & 1821 & 2002 & 1984 & 1928 & 1773 & 2834 & 1754 & 2380 & 848 \\
\hline $\begin{array}{l}\text { Goodness-of-fit } \\
\text { on } F^{2}\end{array}$ & 0.978 & 1.030 & 0.973 & 1.031 & 1.120 & 1.035 & 0.962 & 1.040 & 1.016 & 1.188 \\
\hline $\begin{array}{l}\text { Final } R \text { indices } \\
{[I>2 \sigma(I)]}\end{array}$ & $\begin{array}{l}R_{1}=0.0254 \\
w R_{2}=0.0438\end{array}$ & $\begin{array}{l}R_{1}=0.0357 \\
w R_{2}=0.0677\end{array}$ & $\begin{array}{l}R_{1}=0.0352 \\
w R_{2}=0.0644\end{array}$ & $\begin{array}{l}R_{1}=0.0190 \\
w R_{2}=0.0405\end{array}$ & $\begin{array}{l}R_{1}=0.0209 \\
w R_{2}=0.0490\end{array}$ & $\begin{array}{l}R_{1}=0.0263 \\
w R_{2}=0.0568\end{array}$ & $\begin{array}{l}R_{1}=0.0392 \\
w R_{2}=0.0652\end{array}$ & $\begin{array}{l}R_{1}=0.0255 \\
w R_{2}=0.0491\end{array}$ & $\begin{array}{l}R_{1}=0.0349 \\
w R_{2}=0.0640\end{array}$ & $\begin{array}{l}R_{1}=0.0553 \\
w R_{2}=0.1098\end{array}$ \\
\hline $\begin{array}{l}R \text { indices (all } \\
\text { data) }\end{array}$ & $\begin{array}{l}R_{1}=0.0364 \\
w R_{2}=0.0472\end{array}$ & $\begin{array}{l}R_{1}=0.0360 \\
w R_{2}=0.0681\end{array}$ & $\begin{array}{l}R_{1}=0.0358 \\
w R_{2}=0.0650\end{array}$ & $\begin{array}{l}R_{1}=0.0221 \\
w R_{2}=0.0414\end{array}$ & $\begin{array}{l}R_{1}=0.0242 \\
w R_{2}=0.0502\end{array}$ & $\begin{array}{l}R_{1}=0.0335 \\
w R_{2}=0.0595\end{array}$ & $\begin{array}{l}R_{1}=0.0618 \\
w R_{2}=0.0739\end{array}$ & $\begin{array}{l}R_{1}=0.0352 \\
w R_{2}=0.0521\end{array}$ & $\begin{array}{l}R_{1}=0.0366 \\
w R_{2}=0.654\end{array}$ & $\begin{array}{l}R_{1}=0.0639 \\
w R_{2}=0.1125\end{array}$ \\
\hline $\begin{array}{l}\text { Largest diff. peak } \\
/ \text { hole, } e / \AA^{3}\end{array}$ & $1.098 /-1.303$ & $1.475 /-2.037$ & $1.748 /-2.161$ & $0.442 /-1.116$ & $0.493 /-1.222$ & $0.821 /-1.223$ & $1.784 /-1.695$ & $0.696 /-1.144$ & $1.753 /-1.427$ & $0.983 /-0.947$ \\
\hline
\end{tabular}




\section{Computational details}

The single point calculations based on the experimental X-ray geometries of $\mathbf{1}$ and $\mathbf{2}$ have been carried out at the DFT level of theory using the M06 functional [Theor. Chem. Acc. 2008, 120, 215.] with the help of Gaussian-09 [M. J. Frisch et al., in Gaussian 09, Revision C.01, Gaussian, Inc., Wallingford, CT, 2010.] program package. The Douglas-Kroll-Hess $2^{\text {nd }}$ order scalar relativistic calculations requested relativistic core Hamiltonian were carried out using the DZPDKH basis sets [Mol. Phys. 2010, 108, 1965. II J. Chem. Phys. 2009, 130, 064108. II Chem. Phys. Lett. 2013, 582, 158. II J. Mol. Struct. - Theochem 2010, 961, 107.] for all atoms. The topological analysis of the electron density distribution with the help of the atoms in molecules (QTAIM) method developed by Bader [Chem. Rev. 1991, 91, 893.] has been performed by using the Multiwfn program (version 3.7) [J. Comput. Chem. 2012, 33, 580.]. The Cartesian atomic coordinates for model supramolecular associates are presented in Table S2.

Table S2. Cartesian atomic coordinates for model supramolecular associates.

\begin{tabular}{|c|c|c|c|}
\hline Atom & $X$ & $Y$ & Z \\
\hline \multicolumn{4}{|c|}{1} \\
\hline Sn & 3.265871 & 5.831769 & 6.290825 \\
\hline $\mathrm{Cl}$ & 1.570799 & 7.526841 & 6.290825 \\
\hline $\mathrm{Cl}$ & 3.830925 & 6.335506 & 3.980582 \\
\hline $\mathrm{Cl}$ & 4.985234 & 4.112406 & 6.290825 \\
\hline $\mathrm{Cl}$ & 1.623110 & 4.189827 & 5.503465 \\
\hline $\mathrm{Cl}$ & 2.762134 & 5.266715 & 8.601068 \\
\hline $\mathrm{Cl}$ & 4.907813 & 7.474530 & 7.078185 \\
\hline $\mathrm{Cl}$ & -0.846353 & 2.210908 & 6.034663 \\
\hline $\mathrm{Cl}$ & -2.210908 & 0.846353 & 6.546987 \\
\hline Sn & -5.831769 & -3.265871 & 6.290825 \\
\hline $\mathrm{Cl}$ & -7.526841 & -1.570799 & 6.290825 \\
\hline $\mathrm{Cl}$ & -5.266715 & -2.762134 & 3.980582 \\
\hline
\end{tabular}




\begin{tabular}{|c|c|c|c|}
\hline $\mathrm{Cl}$ & -4.112406 & -4.985234 & 6.290825 \\
\hline $\mathrm{Cl}$ & -7.474530 & -4.907813 & 5.503465 \\
\hline $\mathrm{Cl}$ & -6.335506 & -3.830925 & 8.601068 \\
\hline $\mathrm{Cl}$ & -4.189827 & -1.623110 & 7.078185 \\
\hline \multicolumn{4}{|c|}{2} \\
\hline $\mathrm{Pb}$ & 3.276851 & 5.839949 & 6.292150 \\
\hline $\mathrm{Cl}$ & 5.047790 & 4.069010 & 6.292150 \\
\hline $\mathrm{Cl}$ & 1.531805 & 7.584995 & 6.292150 \\
\hline $\mathrm{Cl}$ & 1.580306 & 4.142856 & 7.107613 \\
\hline $\mathrm{Cl}$ & 2.764305 & 5.256018 & 3.905412 \\
\hline $\mathrm{Cl}$ & 4.973944 & 7.536494 & 5.476687 \\
\hline $\mathrm{Cl}$ & 3.860782 & 6.352495 & 8.678888 \\
\hline $\mathrm{Cl}$ & -0.828170 & 2.187120 & 6.551387 \\
\hline $\mathrm{Cl}$ & -2.187120 & 0.828170 & 6.032913 \\
\hline $\mathrm{Pb}$ & -5.839949 & -3.276851 & 6.292150 \\
\hline $\mathrm{Cl}$ & -4.069010 & -5.047790 & 6.292150 \\
\hline $\mathrm{Cl}$ & -7.584995 & -1.531805 & 6.292150 \\
\hline $\mathrm{Cl}$ & -7.536494 & -4.973944 & 7.107613 \\
\hline $\mathrm{Cl}$ & -6.352495 & -3.860782 & 3.905412 \\
\hline $\mathrm{Cl}$ & -4.142856 & -1.580306 & 5.476687 \\
\hline $\mathrm{Cl}$ & -5.256018 & -2.764305 & 8.678888 \\
\hline
\end{tabular}




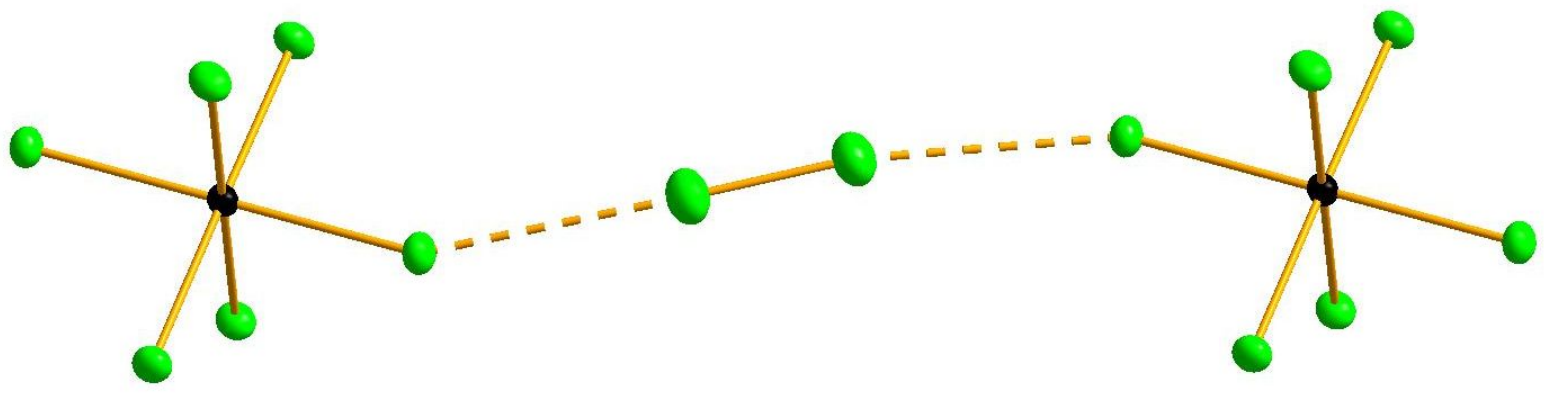

Figure S3. Fragment of the structure of 1 represented in thermal ellipsoids 